\title{
ATRIBUTOS FÍSICOS DO SOLO EM UM SISTEMA SILVIPASTORIL COM NÚCLEOS ARBÓREOS NO ESTADO DE SANTA CATARINA
}

\author{
L. F. Z. BATTISTI ${ }^{1}$, A. L. SCHMITT FILHO ${ }^{2}$, A. LOSS ${ }^{3}$, J. FARLEY ${ }^{4}$ \\ Universidade Federal de Santa Catarina ${ }^{1,2,3}$, University of Vermont, Burlington ${ }^{4}$ \\ ORCID ID: http://orcid.org/0000-0002-3005-6158 \\ arcangelo.loss@ufsc.br ${ }^{3}$
}

Submetido 28/01/2020 - Aceito 16/10/2020

DOI: $10.15628 /$ holos.2020.9473

\section{RESUMO}

Sistemas silvipastoris e o pastoreio racional voisin (PRV) podem elevar a qualidade dos atributos físicos de solos pastoris degradados, aumentando a biodiversidade e a produtividade agrícola. A partir disto, este trabalho teve como objetivo avaliar os efeitos da implantação do sistema silvipastoril com quatro anos com núcleos arbóreos de diversidade (SSPnúcleos) sob os atributos físicos do solo, comparando com pastagens desprovidas de sombra natural (PDS), floresta primária (F1) e floresta secundária (F2). Foram avaliados os seguintes indicadores de qualidade do solo: densidade do solo (Ds) e os índices de agregação do solo: diâmetro médio geométrico (DMG) e o índice de sensibilidade do DMG (ISDMG). Os resultados evidenciaram que os sistemas SSPnúcleos e PDS apresentaram valores superiores de Ds em relação à $\mathrm{F} 1$, mas estes valores são inferiores ao valor crítico para desenvolvimento de raízes. A pesquisa demonstrou o potencial dos tratamentos SSPnúcleos e PDS para a promoção da estruturação física do solo, apresentando valores de DMG e ISDMG superiores à F1, que representa a condição original do solo. Além disso, observou-se que a presença de árvores não influenciou negativamente, tão pouco positivamente nos atributos físicos do solo na região sombreada no entorno dos núcleos, onde os animais se concentram. Dessa forma, foi possível inferir que os manejos agroecológicos (PRV e o SSPnúcleos) promovem o processo de agregação do solo de forma mais eficiente em comparação às áreas de floresta, com destaque para a floresta secundária, evidenciando sua capacidade de restauração da estrutura física do solo.

PALAVRAS-CHAVE: agregação do solo, estrutura física do solo, teoria da nucleação, agricultura biodiversa.

\section{SOIL PHYSICAL PROPERTIES UNDER SILVOPASTORAL SYSTEM WITH DIVERSITY TREE NUCLEI IN SANTA CATARINA STATE}

\section{ABSTRACT}

Conservation farming systems, such as the silvopastoral system and Management intensive grazing (MIG), have been presented as efficient solutions to raise the quality of the physical attributes of poorly managed soils, as well as to leverage local biodiversity and agricultural productivity. Based on this, this work evaluated the effects of silvopastoral system tree nuclei with four-yearold (SPSnuclei) under the physical attributes of the soil, comparing with MIG areas without trees, primary forest (F1) and secondary forest (F2). The following soil quality indicators were evaluated: soil density (SD) and aggregation indices: geometric mean diameter (GMD) and sensitivity index - SIGMD). The results showed that
\end{abstract}

the SPSnuclei and MIG systems presented higher values of SD than F1, but these values are lower than the critical value for root development. In addition, the results evidenced the potential of SPSnuclei and MIG treatments to promote the physical structuring of the soil, presenting values of GMG and SIGMD higher than F1, which represents the original condition of the soil. Thus, it was possible to infer that agroecological management (MIG and SSPnuclei) promote the process of soil aggregation more efficiently compared to forest areas, especially secondary forest, evidencing its ability to restore the physical structure of the soil.

KEYWORDS: Soil aggregation, soil physical structure, nucleation theory, biodiversified agriculture. 


\section{INTRODUÇÃO}

O estado de Santa Catarina (SC), localizado na região Sul do Brasil, é ocupado por 839.136 hectares de áreas com pastagem (MapBiomas, 2020), e é responsável por 8,9\% da produção nacional de leite (EPAGRI, 2019). Grande parte dessas áreas de pastagens são manejadas extensivamente no sistema de manejo de baixa produtividade, caracterizado pela dependência de fertilizantes solúveis, com mobilização anual dos solos para o cultivo monocultural de grãos para a produção de silagem (Alvez et al., 2014). Manejos não-conservacionistas do solo, sob áreas de pastagem, podem promover a degradação da estrutura física, permitir a erosão, reduzir a macroporosidade e a infiltração de água, aumentar a resistência do solo à penetração de raízes, reduzir o volume total de poros do solo e promover erosão laminar (Byrnes et al., 2018; Brizzi et al., 2019). Estes processos acarretam no aumento da densidade do solo (Ds) e no menor desenvolvimento de macroagregados, culminando em uma contínua diminuição da produtividade (Sena et al., 2017). Entretanto, sistemas de manejo conservacionistas podem reverter este processo e melhorar os atributos físicos do solo, tais como a agregação e a Ds (Comin et al., 2016; Martinkoski et al., 2017).

Um exemplo de sistema conservacionista para agroecossistemas pastoris é o sistema Voisin ou Pastoreio Racional Voisin (PRV), que baseia-se na divisão da pastagem em parcelas ou piquetes, sendo desenvolvido na França por André Voisin (Voisin, 1961). Segundo Murphy (1987) e Pinheiro Machado (2010), o PRV pressupõe a manutenção e elevação da fertilidade do solo, a maximização do uso de recursos disponíveis na propriedade, a maior rentabilidade, a produção de alimentos sem uso de agrotóxicos e o respeito ao bem-estar animal. Através do PRV, a produtividade animal e a capacidade de suporte do sistema podem aumentar significantemente, e a incidência de pragas diminuírem sem a necessidade do aumento da área de pastagem O PRV favorece os atributos edáficos através do desenvolvimento uniforme e periódico das raízes das forrageiras, promovendo a descompactação do solo, o desenvolvimento de macroagregados e a infiltração da água, através das galerias abertas pelo crescimento radicular (Murphy et al., 1994; Comin et al., 2016; Battisti et al., 2018; Yadav et al., 2019).

Todavia, apesar do PRV ser uma ótima alternativa, a contenção dos animais nos piquetes, que é necessário para viabilizar o tempo de repouso as forrageiras, impede o livre acesso do rebanho às áreas sombreadas da propriedade nos horários mais quentes do dia. Com isso, a falta de áreas sombreadas na pastagem pode ser solucionada com a implantação de sistemas silvipastoris, caracterizados pela combinação de árvores, animais e pastagem em um mesmo agroecossistema (Bernardino \& Garcia, 2010).

Após participar de um amplo processo de fortalecimento, a agricultura familiar no Sul do Brasil vem implantando mais de 500 projetos de produção de leite agroecológico em sistema Voisin (PRV), o Grupo de Pastoreio Voisin da Universidade Federal de Santa Catarina (GPVoisin/UFSC) resgata uma proposta aparentemente rejeitada durante uma década pelos produtores, 0 
sombreamento das pastagens com árvores nativas (GPVoisin/UFSC, 2007). Este resgate foi motivado por uma demanda pontual da Associação dos Agricultores Ecológicos das Encostas da Serra Geral - Santa Catarina - Brasil (AGRECO), e está relacionada com a saída dos rebanhos dos estábulos e a alimentação eminentemente à base de pasto (Schmitt Filho et al., 2010; Alvez et al., 2014; Battisti et al., 2018). Em 2011/12 os primeiros produtores estavam implantando o Sistema Silvipastoril com Núcleos de diversidade arbórea (SSPnúcleos) idealizado por Schmitt et al. (2010) no Laboratório de Sistemas Silvipastoris e Restauração Ecológica (LASSre/UFSC), antes denominado GPVoisin/UFSC. Após quatro anos de pesquisa-ação participativa (Mendez et al., 2017), o sistema foi idealizado para viabilizar sombra e produtos florestais não madeireiros com espécies nativas já a partir do segundo ano e, sinergicamente, trazer multifuncionalidade para a paisagem e iniciar processos de restauração ecológica do agroecossistema pastoril (Silva et al., 2017; Battisti et al., 2018; Deniz et al., 2019; Simioni et al., 2019).

O SSPnúcleos foi inspirado na teoria de nucleação como princípio sucessional de recuperação de áreas florestais (Reis \& Kageyama, 2003), sendo estruturado em núcleos arbóreos ou ilhas de alta diversidade (Schmitt Filho et al., 2016). O SSPnúcleos se caracteriza por ser um sistema silvipastoril no qual o elemento arbóreo está inserido em 40 núcleos de $25 \mathrm{~m}^{2}$ cada, dispostos equidistantemente por hectare de pastagem. A área de todos os núcleos somados em um hectare totaliza o equivalente a $10 \%$ da área total de pastagem (Schmitt Filho et al., 2013; Schmitt Filho et al., 2016). O SSPnúcleos é um sistema silvipastoril que busca promover a conservação ambiental, utilizando somente espécies nativas da Mata Atlântica, bioma no qual o sistema está inserido. Esta prática origina um substancial incremento na biodiversidade, além de não comprometer a produtividade agrícola. Ademais, o SSPnúcleos aumenta o potencial conservacionista, pois é constituído por mais de 50 espécies de árvores nativas diferentes por hectare, diversas espécies de meliponídeos, gramíneas e leguminosas forrageiras prioritariamente nativas (Schmitt Filho et al., 2013; Schmitt Filho et al., 2017).

Neste sentido, o objetivo deste trabalho foi avaliar os efeitos da implantação do SSPnúcleos, manejados sob sistema de PRV, nos atributos físicos do solo, comparando com áreas de pastagens desprovidas de árvores (PDA), floresta primária (F1) e floresta secundária (F2) adjacentes.

\section{MATERIAIS E MÉTODOS}

\section{1 Área de estudo}

A pesquisa foi conduzida no município de Santa Rosa de Lima, localizado na região Sul do estado de Santa Catarina, Brasil (28 4'20.45"S, 49 9'17.37"O). A cidade está em média a 235 metros de altitude com uma variação em suas cotas altimétricas de 200 a 1.200 metros. Está inserida na zona climática Subtropical Úmida (Cfa) de Köppen, caracterizado por ser clima subtropical, com 
verão quente. As temperaturas são superiores a $22^{\circ} \mathrm{C}$ no verão, com mais de $30 \mathrm{~mm}$ de chuva no mês mais seco. A precipitação anual total é de 1.400 a 1.600 mm (Alvarez et al., 2013). A vegetação original é composta pela Floresta Ombrófila Densa e alguns pontos Floresta Ombrófila Mista (Rezende et al., 2018).

Para o desenvolvimento deste estudo foram selecionadas quatro áreas com usos distintos do solo: de pastagem desprovida de árvores (PDA), sistema silvipastoril com núcleos arbóreos (SSPnúcleos) implantado há quatro anos, floresta secundária (F2), e floresta primária (F1). Todas as áreas estavam posicionadas no terço médio da paisagem, apresentando relevo ondulado (12-15\% de declividade) e altitude de aproximadamente 240 metros. O solo predominante das áreas experimentais é classificado como Cambissolo Háplico (Embrapa, 2018), e a classe textural classificada como francossiltosa, havendo um predomínio da fração silte, sendo observados valores médios em todas as áreas $(0-40 \mathrm{~cm})$ de silte igual a $500 \mathrm{~g} \mathrm{~kg}^{-1}$, de argila igual a $192 \mathrm{~g} \mathrm{~kg}^{-1}$ e $261 \mathrm{~g} \mathrm{~kg}^{-}$ ${ }^{1}$ de areia.

O SSPnúcleos ( $28^{\circ} 4^{\prime} 25.33^{\prime \prime S}, 49^{\circ} 9^{\prime} 22.15^{\prime \prime O}$ ) foi implantado em 2012, apresentando cinco piquetes em uma área total de $9.800 \mathrm{~m}^{2}$, no qual cada piquete tem aproximadamente $1.900 \mathrm{~m}^{2}$. Ambas as áreas de pastagens eram caracterizadas por uma composição florística polifítica (de alta diversidade de espécies forrageiras), com dominância de Axonopus catharinensis e Hemarthria altissima, manejadas no sistema de Pastoreio Racional Voisin desde 1999. A densidade de núcleos arbóreos é de 40 núcleos por hectare, sendo que cada núcleo é composto por quatro indivíduos de Mimosa scabrella, quatro indivíduos de espécies arbóreas melíferas ou frutíferas, oito Euterpe edulis, além de espécies climácicas, como Caesalpinia echinata e Chorisia speciosa. Os indivíduos arbóreos foram plantados em 2012 em covas de aproximadamente 10 × 20 x 20cm de tamanho, nos núcleos previamente cercados com arame eletrificado a $50 \mathrm{~cm}$ de distância das plantas periféricas. Estes piquetes são ocupados por 35 vacas em lactação, seguidas pelo lote de repasse. Esta propriedade conta com dois lotes, e cada lote permanece em cada piquete aproximadamente 12 horas, e o tempo de repouso são trinta dias. Assim o rebanho permanece no piquete um período médio de um dia por mês, com períodos de repouso de aproximadamente trinta dias entre cada ocupação.

Antes da instalação do SSPnúcleos foi realizada uma calagem para elevação do pH para um valor entre 5,5 a 6,0, e nos últimos seis anos, foram aplicados $50 \mathrm{~kg}$ de fertilizantes nitrogenados por hectare anualmente. Além disso, a pastagem é sobressemeada com Avena sativa, Lolium multiflorum, Trifolium pratense e Trifolium repens no início de cada outono desde 1999.

Já a área de pastagem desprovida de espécies arbóreas (PDA) (28 $\left.{ }^{\circ} 4^{\prime} 24.49^{\prime \prime} \mathrm{S}, 49^{\circ} 9^{\prime} 21.90^{\prime \prime} \mathrm{O}\right)$, localiza-se lado a lado do SSPnúcleos apresentando a mesma idade e condições de manejo semelhantes ao SSPnúcleos, diferenciando-se do SSPnúcleos somente por não apresentar os 
núcleos arbóreos. No PDA tem-se uma pastagem polifítica de mais de 50 anos de idade, com dominância de Axonopus catharinensis e Hemarthria altíssima.

Além das áreas de pastagem, há uma área de seis hectares de Mata Atlântica Ombrófila Densa Primária (F1) (28 4'18.05"S, 49 9'19.92"O), composta predominantemente por árvores como Alsophila setosa, Cyathea phalerata, Sloanea spp, Ocotea spp, entre outras. Este tratamento representa a condição original do solo de todas as áreas (testemunha). Há também, adjacente as áreas, um fragmento de 0,4 hectares de Mata Atlântica ombrófila densa em estágio secundário de sucessão com 15 anos de idade (F2) (28 4'26.65"S, 49 9'16.92"O), composta por espécies como Alchornea triplinervia, Nectandra spp, Byrsonima ligustrifolia, entre outras. Esta área anteriormente fora ocupada com sucessivos cultivos anuais convencionais de Zea mays e pastagem.

\subsection{Amostragem e coleta de dados}

Para a coleta das amostras de solo, realizada em fevereiro de 2016, foram abertas trincheiras $(40 \times 40 \times 40 \mathrm{~cm})$ em cada uma das quatro áreas. Foram coletadas amostras indeformadas para avaliação da densidade do solo em diferentes profundidades (0-5, 5-10, 10-20, 20-30 e 30-40 cm) e estabilidade de agregados (0-5, 5-10 e 10-20 cm).

Nas áreas de pastagem desprovida de arvores (PDA), F1 e F2 foram coletadas três amostras indeformadas simples para formar uma composta, com seis repetições compostas para cada área. No tratamento SSPnúcleos, foram coletadas uma amostra indeformada simples em cada uma das quatro faces dos núcleos (Norte, Sul, Leste e Oeste) a uma distância de $2 \mathrm{~m}$ da cerca do núcleo e mais uma amostra simples no centro dos núcleos. Estas cinco amostras coletadas em cada núcleo constituem uma amostra composta que representou o tratamento SSPnúcleos, com seis repetições compostas (Figura 1). Na área do tratamento PDA, as amostras foram coletadas em locais sob condições de pleno sol, na mesma linha dos núcleos da área de SSPnúcleos, onde foram coletadas as amostras, uma vez que os dois tratamentos estão localizados lado a lado. Nas áreas de floresta (F1 e F2) foram coletadas amostras distribuídas aleatoriamente dentro de uma área delimitada de 300 a $400 \mathrm{~m}^{2}$. 


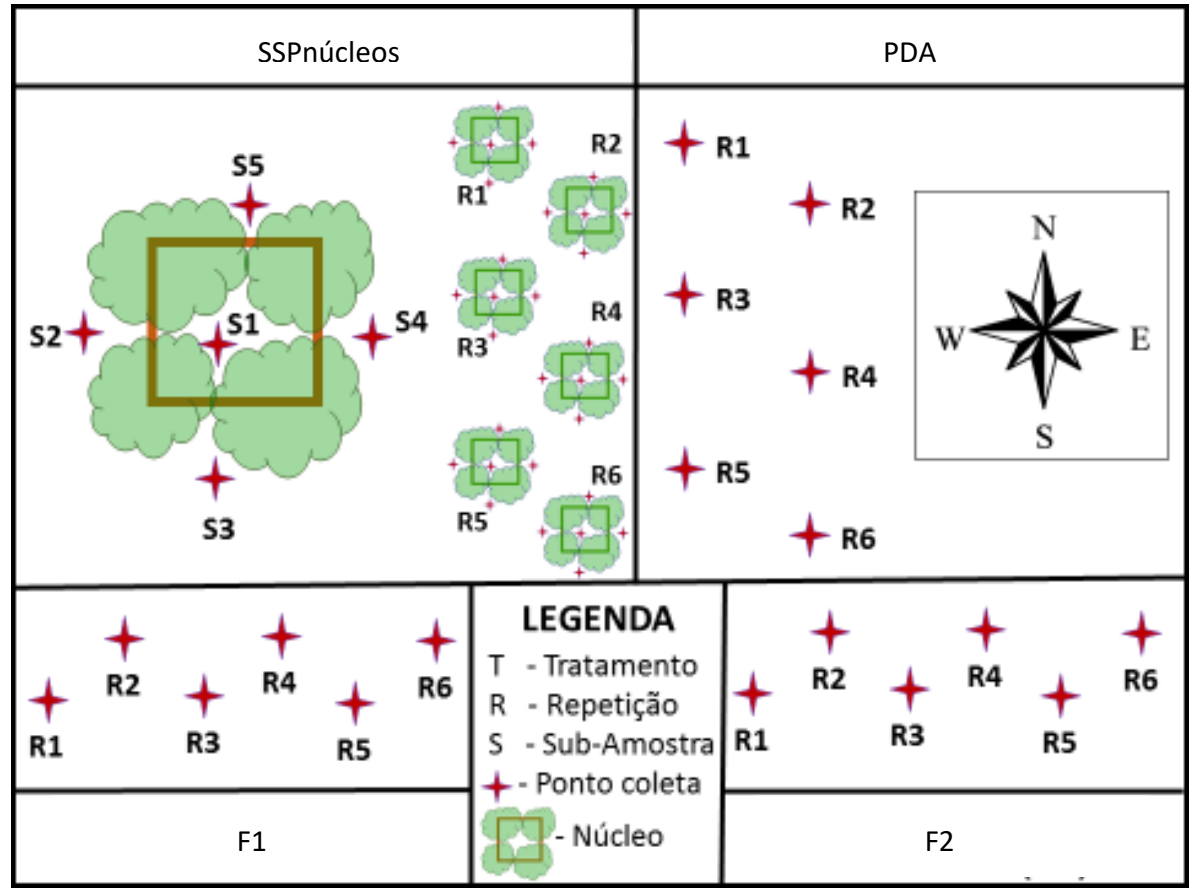

Figura 1: Croqui esquemático das áreas amostradas, com destaque para a disposição dos pontos coletados.

Após a coleta, as amostras foram secas ao ar e processadas no Laboratório de Manejo e Classificação de Solos da Universidade Federal de Santa Catarina para posterior avaliação dos atributos físicos do solo. A densidade do solo (Ds) foi determinada por meio da coleta das amostras indeformadas, no sentindo horizontal, pelo método do anel volumétrico (Kopecky), que possui volume conhecido $\left(50 \mathrm{~cm}^{3}\right)$. A Ds foi calculada pela seguinte equação: $\mathrm{Ds}=\left(\mathrm{g} \mathrm{cm}^{-3}\right)=\mathrm{Ms} / \mathrm{Vs}$; onde $\mathrm{Ms}=$ massa seca do solo, em g; e Vs = volume do solo, em $\mathrm{cm}^{3}$, conforme descrito em Embrapa (2017). A agregação do solo foi avaliada através da distribuição dos agregados estáveis em água, utilizado o método da Embrapa (2017). As amostras indeformadas (torrões) que foram secas à sombra no laboratório, posteriormente foram passadas em um conjunto de peneiras de malha de 8,0 e 4,0 mm, para obtenção dos agregados. Em seguida, foram pesadas $25 \mathrm{~g}$ das amostras dos agregados que ficaram retidos na peneira de $4,0 \mathrm{~mm}$. Posteriormente, as amostras foram transferidas para um jogo de peneiras com malhas de 2,00; 1,00;0,50;0,25 e 0,105 mm, umedecidas com pulverizador manual, sendo submetido à agitação vertical no aparelho de Yoder, durante 15 min. Após o tempo determinado, o material retido em cada peneira foi retirado, separado com o auxílio de jato d'água, colocado em placas de petri, secas em estufa a $105^{\circ} \mathrm{C}$, até atingir massa constante. Após a secagem, obtém-se a massa dos agregados retida em cada peneira. Com os dados de massa seca dos agregados foi calculado o diâmetro médio geométrico (DMG) dos agregados. A partir dos dados de DMG, calculou-se o índice de sensibilidade (Bolinder et al., 1999), utilizando-se a expressão: IS = DMGt / DMGo, em que IS: índice de sensibilidade; DMGt: valor do DMG do solo 
em cada tratamento; e DMGo: valor do DMG do solo na cobertura original (Mata Atlântica primária $-F 1)$.

\subsection{Análises estatísticas}

Os resultados foram analisados quanto à normalidade e homogeneidade dos dados por meio dos testes de Lilliefors e Barttlet, respectivamente. Os dados apresentaram distribuição normal e homogeneidade das variâncias, não sendo necessário realizar nenhuma transformação. Posteriormente, foi analisado como delineamento inteiramente casualizado, pois os sistemas de uso do solo avaliados estão sob as mesmas condições topográficas e edafoclimáticas. Os resultados foram submetidos à análise de variância com aplicação do teste $\mathrm{F}$ e os valores médios, quando significativos, comparados entre si pelo teste de Tukey a $5 \%$ de probabilidade de erro.

\section{RESULTADOS E DISCUSSÃO}

\subsection{Densidade do solo}

Os valores de densidade do solo (Ds) variaram de 0,84 a 1,11 $\mathrm{Mg} \mathrm{m}^{-3}$ na profundidade de 0$5 \mathrm{~cm}$, e de 1,04 a $1,46 \mathrm{Mg} \mathrm{m}^{-3}$, na profundidade de $30-40 \mathrm{~cm}$. Os menores valores de Ds foram encontrados no tratamento F1 em todas as profundidades avaliadas (Figura 2).

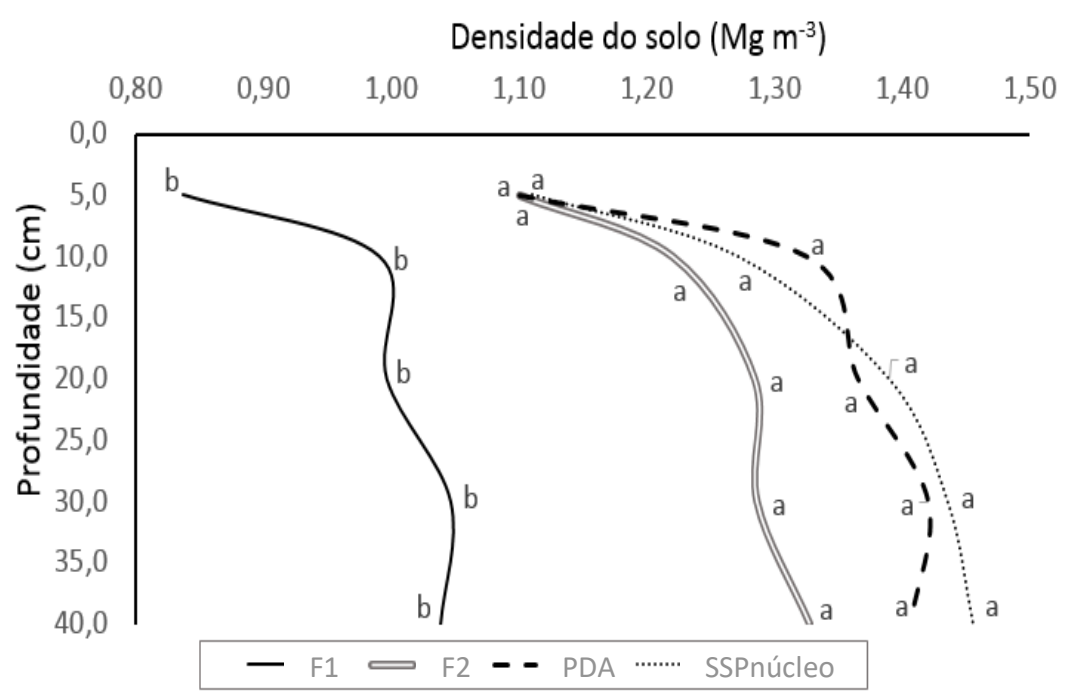

Figura 2: Densidade do solo $\left(\mathrm{Mg} \mathrm{m}^{-3}\right)$ encontrada nos diferentes sistemas de uso do solo em Santa Rosa de Lima, SC. Letras iguais, em cada profundidade, não diferem estatisticamente a $5 \%$ de probabilidade de erro pelo Teste de Tukey. Tratamentos: SSPnúcleos = Sistema silvipastoril com núcleos Arbóreos, PDA = pastoreio racional voisin desprovido de árvore, F2 = Floresta secundária, F1 - Floresta primária. 
Não foram observadas diferenças estatísticas entre os valores de Ds para os tratamentos SSPnúcleos e PDA, o que indica que a implantação dos núcleos arbóreos para o fornecimento de sombra para o gado não influenciou no aumento da Ds, apesar de Craesmeyer et al. (2017) ter observado uma maior concentração do rebanho nas áreas adjacentes aos núcleos. Embora dos tratamentos SSPnúcleos e PDA apresentarem os maiores valores de densidade, é importante ressaltar que todos os valores de Ds encontrados são inferiores a $1,55 \mathrm{Mg} \mathrm{m}^{-3}$, valor crítico para o desenvolvimento normal das raízes em solos de textura média (Reichert et al., 2003).

O fato dos núcleos SSPnúcleos estarem dispostos equidistantemente em cada piquete, promovendo zonas sombreadas independentes, evita a concentração do rebanho em um único ponto, e uma possível compactação do solo que foi observado em sistemas silvipastoris dispostos em linha (Bezkorowajnyj et al., 1993). Além disso, outro fator que contribui para a não compactação do solo é o manejo rotativo dos piquetes que acontece há 16 anos, que possibilita um período de repouso médio de trinta dias em cada piquete, promovendo o desenvolvimento pleno das raízes da pastagem, dinâmica que atua como um mecanismo contra a compactação do solo.

Entretanto, a ausência de diferenças entre os valores de Ds entre SSPnúcleos e PDA pode ser fruto da pouca idade do sistema no momento deste estudo (2012-2016). Espera-se que este cenário possa mudar com o tempo, sendo necessários mais estudos nestas áreas com a evolução do sistema. Sendo assim, infere-se que o SSPnúcleos mais maduro possa apresentar redução dos valores de Ds em comparação ao PDA, pois no SSPnúcleos já há um aumento do conteúdo de carbono orgânico em comparação ao PDA (Battisiti et al., 2018). Avaliando a Ds em áreas de PRV de cinco anos com e sem árvores dispostas na pastagem e em uma floresta nativa, Comin et al. (2016) também não encontraram diferenças estatísticas entre os sistemas de PRV com e sem árvores nas camadas de 05, 5-10 e 10-20 cm, com valores variando entre 0,93 a 1,11 Mg.m-3. Os autores também encontraram maiores valores de Ds nas áreas de PRV em comparação à floresta, sendo estes resultados semelhantes ao deste estudo.

Analisando os valores de Ds nas profundidades de $0-20 \mathrm{~cm}, 20-40 \mathrm{~cm}$ e $40-60 \mathrm{~cm}$ entre um SSP composto por uma vegetação arbórea disposto aleatoriamente e uma área de floresta secundária com 20 anos desde que foi isolada da área na qual hoje é o SSP, Martinkoski et al. (2017) não encontraram diferenças estatísticas entre os dois tratamentos em nenhuma profundidade. $O$ mesmo foi observado neste estudo quando comparamos SSPnúcleos e PDA.

Os menores valores de Ds encontrados no tratamento F1 representam a tendência natural de solos de florestas em apresentarem baixos valores de Ds, pois em ambientes florestais, o solo sofre menos distúrbios do que solos sob usos agrícolas, que são perturbados com o tráfego de pessoas, animais ou máquinas, que promovem o aumento da Ds (Bezkorowajnyj et al., 1993; Tanner \& Mamaril, 1959). 
Resultados semelhantes também foram encontrados por Nicodemo et al. (2018), que também compararam atributos físicos do solo entre mata, sistema silvipastoril e pastagem sem árvores, no qual a Ds nas profundidades de $20-40 \mathrm{~cm}$ foi superior estatisticamente nas áreas de pastagem sem árvores e na área do Sistema silvipastoril do que na área de mata.

\subsection{Agregação do solo}

Para o atributo diâmetro médio geométrico (DMG) não foi verificado diferenças estatísticas entre os tratamentos nos primeiros $10 \mathrm{~cm}$ de profundidade, sendo que todos os valores encontrados são maiores que $4,00 \mathrm{~mm}$, indicando boa agregação em todos os tratamentos, pois o máximo valor, segundo o método utilizado (Embrapa, 2017), seria 5,00 mm. Na profundidade de $10-20 \mathrm{~cm}$, as áreas de florestas (F2 e F1) apresentaram os menores valores de DMG, com destaque para os maiores valores encontrados nos tratamentos SSPnúcleos e PDA (Tabela 1).

Tabela 1: Diâmetro médio geométrico (DMG) dos agregados encontrados nos diferentes sistemas de uso do solo em Santa Rosa de Lima, SC.

\begin{tabular}{|c|c|}
\hline Sistemas Avaliados & $\mathrm{DMG}(\mathrm{mm})$ \\
\hline \multicolumn{2}{|c|}{$0-5 \mathrm{~cm}$} \\
\hline SSPnúcleos & $4,580^{\mathrm{ns}}$ \\
\hline PDA & 4,748 \\
\hline $\mathrm{F} 2$ & 4,510 \\
\hline F1 & 4,318 \\
\hline CV (\%) & 8,28 \\
\hline \multicolumn{2}{|c|}{$5-10 \mathrm{~cm}$} \\
\hline SSPnúcleos & $4,448^{n s}$ \\
\hline PDA & 4,656 \\
\hline $\mathrm{F} 2$ & 4,168 \\
\hline F1 & 4,383 \\
\hline CV (\%) & 6,65 \\
\hline \multicolumn{2}{|c|}{$10-20 \mathrm{~cm}$} \\
\hline SSPnúcleos & $4,066 a b$ \\
\hline
\end{tabular}

HOLOS, Ano 36, v.6, e9473, 2020 
PDA

F2

F1

$\mathrm{CV}(\%)$
$4,280 \mathrm{a}$

$3,016 \mathrm{c}$

$3,390 \mathrm{bc}$

16,74

Letras iguais na coluna não diferem estatisticamente a $5 \%$ de probabilidade de erro pelo Tukey; ns= não significativo a $5 \%$ de probabilidade de erro pelo teste F; CV= Coeficiente de Variação. Tratamentos: SSPnúcleos = Sistema silvipastoril com núcleos arbóreos, PRV = Pastoreio racional voisin, F2 = Floresta secundária, F1 - Floresta primária.

As raízes fasciculadas das gramíneas, que são densas e estão bem distribuídas no solo tanto em superfície quanto em profundidade naturalmente favorecem o processo de formação de agregados maiores e mais estáveis nas pastagens (Torres et al., 2013). Isto explica os maiores valores de DMG nos tratamentos PDA e SSPnúcleos, justamente os dois tratamentos que apresentam alta concentração de gramíneas. Por causa da presença das raízes de gramíneas nos tratamentos PDA e SSPnúcleos, o valor de DMG nestes tratamentos são maiores que F1 $(10-20 \mathrm{~cm})$, tratamento que mostra as condições naturais do solo, evidenciando que este tipo de manejo promove a formação de agregados maiores e mais estáveis. Além disso, nestes tratamentos há o aporte constante de dejetos bovinos, o que favorece o aumento do conteúdo de matéria orgânica (Battisti et al., 2018) e, consequentemente, também favorece a formação de agregados de maior tamanho (Denef et al., 2007).

O menor valor de DMG encontrado em F2 na profundidade de $10-20 \mathrm{~cm}$ provavelmente é resultado do manejo do solo desenvolvido anteriormente ao restabelecimento da sucessão florestal, caracterizado por cultivos anuais e constante revolvimento do solo. Resultados semelhantes aos encontrados por Loss et al. (2014).

Os resultados de agregação obtidos nesta pesquisa contrastam com os valores encontrados por Neves et al. (2007), que em uma pesquisa desenvolvida em uma área de sistema silvipastoril composto por eucalipto consorciado com pastagem Brachiaria brizantha, manejado sob pastoreio contínuo e extensivo por mais de 20 anos. Os autores não encontraram diferenças estatísticas nos valores de DMG nos primeiros $20 \mathrm{~cm}$ de solo entre as áreas de sistema silvipastoril, pastagem sem árvores e à área de cerrado nativo. Diferente do resultado obtido nesta pesquisa, no qual os valores de DMG são superiores nos tratamentos PDA e SSPnúcleos em relação à F1 na camada de $10-20 \mathrm{~cm}$. Porém, deve-se atentar para o fato de que os dois estudos apresentam condições edafoclimáticas, manejo e composição florística diferentes, tornando importante ressaltar que em cada situação deve-se compor e estudar diferentes espécies e formas de manejo, de forma a definir qual a melhor forma a ser utilizada em cada situação edafoclimática. 
Quanto ao índice de sensibilidade para o DMG (IS $\left.S_{D M G}\right)$ é possível observar valores superiores aos da área referência (F1) para os tratamentos PDA e SSPnúcleos em todas as profundidades. Os valores acima da unidade (área de floresta primária $=1,0$ ), encontrados nas áreas de SSPnúcleos e PDA representam incremento na agregação, e valores menores que a unidade, representam redução na agregação (Bolinder et al., 1999). Para a área F2 verificou-se uma redução do IS de $5 \% \mathrm{e}$ $11 \%$, respectivamente, para 5-10 e 10-20cm, em relação à F1. Já para as áreas de PDA e SSPnúcleos, verificaram-se aumento do IS de $10 \%$ e $6 \%(0-5 \mathrm{~cm})$ e $26 \%$ e $20 \%(10-20 \mathrm{~cm})$, respectivamente, em relação a F1 (Tabela 2).

Tabela 2: Índices de Sensibilidade para o parâmetro diâmetro médio geométrico (ISDMG) de agregados em relação à área referência (floresta primária - F1) nos diferentes sistemas de uso do solo em Santa Rosa de Lima, SC.

\begin{tabular}{lllll}
\hline Prof. $(\mathrm{cm})$ & SSPnúcleos & PDA & F2 & F1 \\
\hline $0-5$ & 1,06 & 1,10 & 1,05 & 1,00 \\
$5-10 \mathrm{Cm}$ & 1,02 & 1,06 & 0,95 & 1,00 \\
$10-20 \mathrm{Cm}$ & 1,20 & 1,26 & 0,89 & 1,00 \\
\hline
\end{tabular}

Tratamentos: SSPnúcleos = Sistema silvipastoril com núcleos arbóreos, PRV = Pastoreio acional voisin, F2 = Floresta secundária, F1 - Floresta primária. Prof = profundidade

Os maiores incrementos do ISDMG observados nas áreas de SSPnúcleos e PDA, com destaque para a profundidade de $10-20 \mathrm{~cm}$, são decorrentes dos maiores valores de DMG encontrados nestas áreas (Tabela 1). Nas profundidades de $0-5$ e $5-10 \mathrm{~cm}$, para os valores de DMG não foram encontradas diferenças entre as áreas avaliadas (Tabela 1), porém quando se observa o IS DMG, notase que as áreas de PDA e SSPnúcleos apresentam incremento no IS em relação a F1 e também em comparação a F2 (Tabela 2). Na PDA e SSPnúcleos, os maiores incrementos no IS estão relacionados à presença das gramíneas, pois suas densas raízes promovem a formação de agregados maiores, o que é acentuado com o manejo do PRV, que permite o repouso do piquete e o consequente desenvolvimento das raízes após a diminuição da massa de raízes em função da desfolhação do pastoreio mensal de cada piquete.

\section{CONCLUSÃO}

A implantação dos núcleos arbóreos em pastagens manejadas sob sistema Voisin não influenciou na densidade do solo quando comparado ao PRV sem núcleos. Cabe destacar que nenhum valor de densidade do solo encontrado neste estudo interfere no desenvolvimento normal das raízes. Além disso, através da análise dos atributos diâmetro médio geométrico e índice de 
sensibilidade foi possível inferir que os manejos agroecológicos (PRV e o SSPnúcleos) promovem o processo de agregação do solo de forma mais eficiente em comparação às áreas de floresta, com destaque para a floresta secundária, evidenciando sua capacidade de restauração da estrutura física do solo.

\section{REFERÊNCIAS BIBLIOGRÁFICAS}

Alvez, J. P.; Schmitt, A. L.; Farley, J. C.; Erickson J. D.; Méndez V. E. (2014). Transition from SemiConfinement to Pasture-Based Dairy in Brazil: Farmers' View of Economic and Environmental Performances. Agroecology and Sustainable Food System, 38(9), 995-1014. doi:10.1080/21683565.2013.859222

Alvares, C.A.; Stape, J.L.; Sentelhas, P.C.; Gongalves, J.L.M.; Sparovek, G. (2013). Koppen's climate classification map for Brazil. Meteorologische Zeitschrift. 22, 711-728. doi.org/10.1127/09412948/2013/0507

Battisti, L. F. Z.; Schmitt Filho, A. L.; Loss, A.; Sinisgalli, P. A. D. A. (2018). Soil chemical attributes in a high biodiversity silvopastoral system. Acta Agronómica, 67(4), 486-493. doi:10.15446/acag.v67n4.70180

Bernardino, F.; Garcia, R. (2010). Sistemas silvipastoris. Pesquisa Florestal Brasileira, 60, 77-87. Disponível em http://www.cnpf.embrapa.br/pfb/index.php/pfb/article/viewArticle/48.

Bezkorowajnyj, P. G.; Gordon, A. M.; Mcbride, R. A. (1993). The effect of cattle foot traffic on soil compaction in a silvo-pastoral system. Agrofororestry System, 21(1), 1-10, doi:10.1007/BF00704922

Byrnes, R. C., Eastburn, D. J., Tate, K. W., Roche, L. M. (2018). A global meta-analysis of grazing impacts on soil health indicators. Journal of environmental quality, 47(4), 758-765. doi/epdf/10.2134/jeq2017.08.0313

Bolinder, M .A.; Angers D. A.; Gregorich, E. G. (1999). The response of soil quality indicators to conservation management. Canadian Journal of Soil Science. 79, 37-45. doi/abs/10.4141/S97$\underline{099}$.

Brizzi, R. R., Portocarrero, H., Costa, N. M. C., Souza, A. P., Costa, A. J. S. T. (2019). Análise Das Características Físico-Químicas Do Horizonte Superficial De Um Latossolo Amarelo Sob Pastagem Como Subsídio À Compreensão De Processos Erosivos No Município De ParatyRj. Caminhos De Geografia, 20(69), 223-236.

Comin, J.; Bourscheid, C.; Souza, M.; Sepulveda, C. M; Loss, A. (2016). Avaliação Da Arborização De Pastagens Em Pastoreio Racional Voisin Por Meio De Indicadores Quantitativos De Qualidade 
Do Solo. Cadernos De Agroecologia. 10(3), 1-5, Disponível Em: Http://Revistas.AbaAgroecologia.Org.Br/Index.Php/Cad/Article/View/17556.

Craesmeyer, K. C.; Schmitt Filho, A. L.; Hotzel, M. J.; Diniz, M.; Farley, J. (2017). Utilização Da Sombra Por Vacas Lactantes Sob Sistema Voisin Silvipastoril No Sul Do Brasil. Cadernos De Agroecologia, 11(2), 1-5. Disponível Em: Http://Revistas.AbaAgroecologia.Org.Br/Index.Php/Cad/Article/View/21524/12572

Denef, K.; Zotarelli, L.; Boddey, R.; Six, J. (2007). Microaggregate-associated carbon as a diagnostic fraction for management-induced changes in soil organic carbon in two Oxisols. Soil Biology and Biochemistry, 39(5), 1165-1172. Doi:S0038071706005360

Deniz, M.; Schmitt Filho, A. L.; Farley, J.; De Quadros, S. F.; Hötzel, M. J. (2019). High Biodiversity Silvopastoral System As An Alternative To Improve The Thermal Environment In The Dairy Farms. International Journal Of Biometeorology, 63(1), 83-92. Doi:10.1007/S00484-018-1638-8

EMBRAPA. Sistema brasileiro de classificação de solos. (2018). Centro Nacional de Pesquisa de Solos. 5a ed. Rio Janeiro.

EMBRAPA. Manual de métodos de análise de solo. (2017). 3a ed. Rio de Janeiro.

EPAGRI. Números da agropecuária catarinense - 2019. Florianópolis, SC, (2019). 65p. (Epagri. Documentos, 291) Valor da produção; área plantada; estrutura fundiária.

GPVoisin. 2007. Pastoreio Voisin Da UFSC Mostra As Vantagens De Um Manejo Racional Dos Pastos. Disponível em: <https://noticias.ufsc.br/2007/05/pastoreio-voisin-da-ufsc-mostra-asvantagens-de-um-manejo-racional-dos-pastos/> [Accessado em 16 Septembro 2020].

Loss. A.; Ribeiro, E.; Pereira, M.; Costa, E. (2014). Atributos físicos e químicos do solo em sistemas de consórcio e sucessão de lavoura, pastagem e silvipastoril em Santa Teresa, ES. Bioscience Journal, 30(5), 1347-1357. Disponível em: http://www.seer.ufu.br/index.php/biosciencejournal/article/view/22079.

MAPBIOMAS, Projeto. (2020). Coleção v3. 1 da Série Anual de Mapas de Cobertura e Uso de Solo do Brasil. Disponível: http://mapbiomas. org. Acesso, v. 22.

Martinkoski, I.; Vogel G.F.; Jadoski, S. O. (2017). Qualidade Física do Solo Sob Manejo Silvipastoril e Floresta Secundária. Floresta e Ambiente, 24, 15-25. doi:10.1590/2179-8087.028216.

Méndez, V.E.; Caswell, M.; Gliessman, S. R.; Cohen, R. (2017). Integrating agroecology and participatory action research (PAR): lessons from Central America. Sustainability, 9, 1-19. doi: https://doi.org/10.3390/su9050705

Murphy, B. (1987). Greener Pastures on Your Side of the Fence: Better Farming with Voisin Grazing 
Management. Colchester.

Murphy, B. (1994). Greener pastures on your side of the fence: better farming with Voisin management intensive grazing. Arriba Pub.

Neves, C.D., Silva, M. L. N.; Curi, N.; Cardoso, E. L.; Macedo, R. L. G.; Ferreira, M. M.; Souza, F. D. (2007). Atributos indicadores da qualidade do solo em sistema agrossilvopastoril, no noroeste do Estado de Minas Gerais. Scientia Forestalis. 1(74), 45-53. Disponível em: https://www.ipef.br/publicacoes/scientia/nr74/cap05.pdf.

Nicodemo, M. L. F; Borges, W. L. B.; Souza, I. M. D. (2018). Atributos físicos do solo em quatro sistemas de uso da terra em São Carlos, SP. Revista Brasileira de Ciências Agrárias, 13(2), 1-7. doi:10.5039/agraria.v13i2a5524.

Pinheiro Machado, L. C. (2010). Pastoreio Racional Voisin: Tecnología Agroecológica Para o 30 Milenio. São Paulo.

Reichert, J. M.; Reinert, D. J.; Braida, J. A. (2003). Qualidade dos Solos e Sustentabilidade De Sistemas Agrícolas. Ciência Ambiental. 27(1),20. doi:10.1590/2179-8687.028216

Reis, A.; Kageyama, P.Y. (2003). Restauração de Áreas Degradadas Utilizando Interações Interespecíficas. In: Restauração ecológica de ecossistemas naturais. Fundação de Estudo e Pesquisas Agrícolas e Florestais; Botucatu, 340p.

Rezende, C. L., Scarano, F. R., Assad, E. D., Joly, C. A., Metzger, J. P., Strassburg, B. B. N., Mittermeier, R. A. (2018). From hotspot to hopespot: An opportunity for the Brazilian Atlantic Forest. Perspectives in ecology and conservation, 16(4), 208-214. doi.org/10.1016/j.pecon.2018.10.002

Simioni, G. F., Schmitt Filho, A. L., Joner, F., Fantini, A. C., Farley, J., Moreira, A. T. (2019). Variação da assembleia de aves em áreas pastoris e remanescentes florestais adjacentes. Revista de Ciências Agrárias, 42(4), 884-895. doi.org/10.19084/rca.17601

Silva, A. D. A., Schimit Filho, A. L., Fantini, A. C., Zambiazi, D. C., Sinisgalli, P. A. (2018). Estimativas de biomassa e carbono em sistema silvipastoril com núcleos arbóreos (PRVnúcleos). Cadernos de Agroecologia, 13(1), 1-7. Disponivel em: http://cadernos.abaagroecologia.org.br/index.php/cadernos/article/view/1742

Schmitt Filho, A. L., Fantini, A. C., Farley, J., Sinisgalli, P. (2017). Nucleation theory inspiring the design of High Biodiversity Silvopastoral System in the Atlantic Forest Biome: ecological restoration, family farm livelihood and agroecology. In World Conference on Ecological Restoration. Foz do Iguaçu PR (p. 450).

Schmitt, F. A., Murphy, W., Farley, J. (2010). Grass based agroecologic dairying to revitalize small 
family farms throughout student technical support: The development of a participative methodology responsible for 622 family farm projects. Advances in Animal Biosciences, 1(2), 517-518. Doi.org/10.1017/S2040470010001329

Schmitt Filho, A. L.; Farley, J.; Sinisgali, P.; Fantini, A. C.; Cazella, A. (2016). Payment for Ecosystem Services in Santa Rosa de Lima: Innovative practices to leverage social change and environmental recovery. In: 4th Convención Internacional Agrodesarrollo 2016 \& 11th International Workshop Trees and Shrubs in Livestock Production. Varadero.

Schmitt, A.; Farley, J.; Alvez, J.; Alarcon, G.; Rebollar, P. M. (2013). Integrating agroecology with payments for ecosystens services in Santa Catarina's Atlantic Forest. Governing the Provision of Ecosystens Services, 481, 45-67. doi:10.1007/978-94-007-5176-7_17

Sena, K. N., Maltoni, K. L., Faria, G. A., Cassiolato, A. M. R. (2017). Organic carbon and physical properties in sandy soil after conversion from degraded pasture to Eucalyptus in the Brazilian Cerrado. Revista Brasileira de Ciência do Solo, 41:e0150505. doi.org/10.1590/18069657rbcs20150505

Soldá, C. C.; Comin, J. J; Feistauner, D.; Fabiane, K. C.; Berwanger, A.; Couto, R. R. (2014). Avaliação da Sustentabilidade em Pastagens. Revista Brasileira de Agroecologia, 9(1), 86-91. Disponível em: file://C:/Users/Pos\%20Agroecos/Downloads/12998-1-63849-1-10-20140428.pdf.

Torres, J. L. R.; Rodrigues Junior, D. J.; Vieira, D. M. S. (2013). Alterações nos atributos físicos do solo em função da irrigação e do pastejo rotacionado. Irriga, 18(3), 558-571. Disponível em: https://www.researchgate.net/profile/Jose_Luiz_Torres/publication/287953177_Changes_in_ physical_characteristics_of_soil_as_a_function_of_irrigation_and_rotational_grazing/links/56 9d3f4a08aed27a702f9a45/Changes-in-physical-characteristics-of-soil-as-a-function-ofirrigation-and-rotational-grazing.pdf

VOISIN A. (1961). Grass Productivity. Paris: Island Press.

Yadav, A.; Gendley, M. K.; Sahu, J.; Patel, P. K.; Chandraker, K.; Dubey, A. (2019). Silvopastoral System: A Prototype Of Livestock Agroforestry. The Pharma Innovation Journal, 8(2) 76-82. doi.org/10.1016/j.jclepro.2013.06.020

\section{COMO CITAR ESTE ARTIGO:}

Battisti, L. F. Z., Schmitt Filho, A. L., Loss, A., Farley, J. (2020). Atributos físicos do solo em um sistema silvipastoril com núcleos arbóreos no estado de santa catarina. Holos. 36(6), 1-16.

\section{SOBRE OS AUTORES}

\section{LUIZ FERNANDO ZIN BATTISTI}

Doutorando do Programa de Pós-graduação em Agroecossistemas, Universidade Federal de Santa Catarina, 
Florianópolis, SC. E-mail: $\underline{\text { fernandobattisti@ hotmail.com }}$

ORCID ID: https://orcid.org/0000-0001-8321-0182

\section{ABDON LUIZ SCHMITT FILHO}

Professor do Programa de Pós-graduação em Agroecossistemas, Universidade Federal de Santa Catarina, Florianopolis, SC. E-mail: abdonfilho@ hotmail.com

ORCID ID: https://orcid.org/0000-0002-3553-7727

\section{ARCÂNGELO LOSS, JOSHUA FARLEY}

Professor do Programa de Pós-graduação em Agroecossistemas, Universidade Federal de Santa Catarina, Florianopolis, SC. E-mail: arcangelo.loss@ufsc.br

ORCID ID: http://orcid.org/0000-0002-3005-6158

\section{JOSHUA FARLEY}

Professor (Community Development and Applied Economics) Employment. University of Vermont, Burlington, Estados Unidos. E-mail: joshua.farley@uvm.edu

ORCID ID: https://orcid.org/0000-0002-5793-5240

Editor(a) Responsável: Francinaide de Lima Silva Nascimento

Pareceristas Ad Hoc: RUDINEI DE MARCO E JOSÉ ALVES

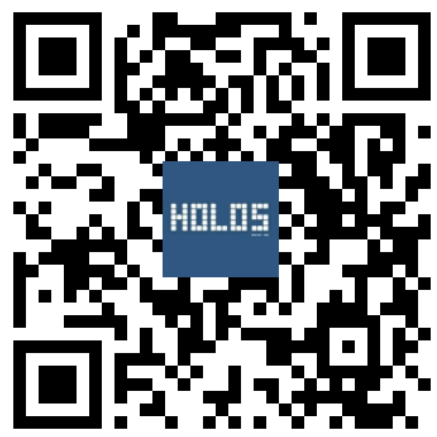

